\title{
Erratum to: Malaria parasite clearance
}

CrossMark

\author{
Nicholas J. White* (1)
}

\section{Erratum to: Malar J (2017) 16:88 \\ DOI 10.1186/s12936-017-1731-1}

After publication of the original article [1], it came to the author's attention that there was an error in the caption of Fig. 6. In the second sentence, the descriptions of the upper and lower panels of the Figure were incorrectly reversed. The correct sentence is as follows:

"The lower panel shows data from areas unaffected by artemisinin resistance, the upper panel shows data from areas where artemisinin resistance is prevalent."

In addition, there was an error in the 'Modelling parasite clearance' section. In the 2nd paragraph, this sentence was incorrect:

"Thus, if a drug exceeded parasiticidal concentrations for $8 \mathrm{~h}$ (e.g. artemisinins) each day and this resulted in a 10,000-fold reduction in parasite density per asexual cycle, then it could be reasoned that ensuring the drug was present continuously at concentrations above the MPC would result in a $10^{12}$ fold reduction per day (i.e. $\left.10^{4} \times 10^{4} \times 10^{4}\right)$."
The sentence should have read as follows:

"Thus, if a drug exceeded parasiticidal concentrations for $8 \mathrm{~h}$ (e.g. artemisinins) each day and this resulted in a 10,000 -fold reduction in parasite density per asexual cycle (i.e. 100 fold per day), then it could be reasoned that ensuring the drug was present continuously at concentrations above the MPC would result in a $10^{6}$ fold reduction per day (i.e. $100 \times 100 \times 100)$."

Published online: 10 May 2017

\author{
Reference \\ 1. White NJ. Malaria parasite clearance. Malar J. 2017;16:88. doi:10.1186/ \\ s12936-017-1731-1.
}

*Correspondence: nickw@tropmedres.ac

Mahidol Oxford Tropical Medicine Research Unit, Faculty of Tropical

Medicine, Mahidol University, 420/6 Rajvithi Road, Bangkok 10400,

Thailand

The online version of the original article can be found under doi:10.1186/

s12936-017-1731-1.
(0 The Author(s) 2017. This article is distributed under the terms of the Creative Commons Attribution 4.0 International License (http://creativecommons.org/licenses/by/4.0/), which permits unrestricted use, distribution, and reproduction in any medium, provided you give appropriate credit to the original author(s) and the source, provide a link to the Creative Commons license, and indicate if changes were made. 